\title{
Investigation of the Freezing/Melting Process of Unsaturated Cement Paste by Low Temperature Calorimetry
}

\author{
Ruihu Zhu \\ College of Harbour Coastal and Offshore Engineering, Hohai University \\ Lin Liu \\ College of Civil and Transportation Engineering, Hohai University \\ Huisu Chen \\ Jiangsu Key Laboratory of Construction Materials, School of Materials Science and Engineering, \\ Southeast University
}

\begin{abstract}
Low temperature calorimetry (LTC) is usually applied to characterize the porosity of cement-based materials with a total water saturated state. By recording the heat flow of the sample at different testing temperatures, the ice content in the freezing and the melting process can be analyzed. In order to investigate the state of the water during the freezing/melting process of unsaturated porous materials, different from previous study, cement pastes of different moisture content are utilized and tested by LTC test. It is found that the nucleation temperature is independent of the degree of saturation in the range of $87 \%-100 \%$, while the influence of the degree of saturation on the melting process is significant. For the melting process, the trough temperature increases with the decreasing degree of saturation, and water movement takes place in unsaturated samples during a freezing/melting cycle.
\end{abstract}

\section{INTRODUCTION}

Low temperature (micro-)calorimetry (LTC) can be applied to characterize the porosity of cementbased materials through an exothermic process (i.e., the freezing process of water) and an endothermic process (i.e., the melting process) (Jiang, Li, Deng, \& Yan, 2013; Sun \& Scherer, 2010; Wu, 2014, 2015; Yamamoto, Endo, Inagi, Ohmori, \& Nakaiwa, 2005). The LTC instrument (calorimeter) records the heat flow of the sample at different testing temperatures and from which the ice content can be calculated by using the heat of fusion for the confined water/ ice in the freezing or the melting process (Wu, 2014, 2015). Different from other porosity characterization methods, such as mercury intrusion porometry (MIP) and nitrogen adsorption/desorption (NAD), the sample for LTC is normally required to be fully saturated with water but not drying treatment. Because the volume of the ice formed/melted under different temperatures indicates the pore information of the sample under the testing. Based on a unique relation between the pore size and the freezing or the melting temperature by thermodynamics, the porosity with different pore sizes can be characterized through the calculated volume of the ice formed/melted under different temperatures.

To realistically reveal the porosity characteristics, the samples have to be totally water saturated (Jiang et al., 2013; Sun \& Scherer, 2010; Yamamoto et al., 2005).
Wu and Johannesson compared the LTC results of samples of different moisture content and indicated that compared with the situation when the pore is fully saturated, the freezing/melting point of the pore water/ ice is lower when the pore is not fully saturated. Hence, it is not appropriate to derive the pore size distribution in the context of LTC analysis using non-fully saturated samples. In this paper, unsaturated cement paste is utilized not only for determining the pore size distribution but also for revealing the state of the water during the freezing/melting process of unsaturated porous materials. Electrical resistance measurements on cement pastes of different moisture content under cyclic freeze-thaw $(\mathrm{F}-\mathrm{T})$ action indicated that water redistribution occurs during freeze-thaw cycles (Wang, Zeng, Wang, Yao, \& Li, 2014). By using LTC analysis on cement pastes of different moisture content, this paper is going to investigate the freezing and the melting processes of unsaturated cement paste where air/water/ice coexist in pores.

\section{MATERIALS AND METHODS}

\subsection{Sample preparation}

Cement paste of water-to-cement ratio (w/c) 0.4 was cast into $8-\mathrm{mm}$ diameter plastic vials and shaped into cylinders after mixing. After curing under sealed condition for 1.5 years, the cement paste was sawn into 
small cylinders with the length of $10 \mathrm{~mm}$. The cylindrical specimens were prepared to have different degrees of saturation before LTC testing. The specimens were oven dried at $105^{\circ} \mathrm{C}$ until constant mass. Then, the specimens were placed in a desiccator and evacuated to a residual pressure of $-7,000 \mathrm{~Pa}$ for $3 \mathrm{~h}$. After evacuation and while still under vacuum, limewater was introduced into the desiccator to cover the specimens. The specimens were left in the desiccator for $18 \mathrm{~h}$. This condition was considered as saturated condition (i.e., 100\% degree of saturation). Some specimens were placed into another desiccator for drying where desiccant was also put in. During the drying period, the mass of the specimens was closely monitored. Specimens were taken out of the desiccator until the degree of saturation $93 \%, 89 \%$, and $87 \%$ was, respectively, achieved. The degree of saturation $S_{w}$ was calculated according to,

$$
S_{w}=\frac{m_{1}-m_{0}}{m_{2}-m_{0}}
$$

where $m_{0}, m_{1}$, and $m_{2}$, respectively, represent the weight of samples after oven dried at $105^{\circ} \mathrm{C}$, after vacuum saturated, and after drying in the desiccator for a period of time.

The specimens of different degrees of saturation were crashed into small pieces of tens of milligrams. Drops of kerosene were added on the piece-like samples to prevent water evaporation in the air. LTC tests were performed on the piece-like samples of different degrees of saturation, i.e., $100 \%, 93 \%, 89 \%$, and $87 \%$, and designated as S100, S93, S89, and S87. The temperature cycle ranged from $+20^{\circ} \mathrm{C}$ to about $-25^{\circ} \mathrm{C}$ at a rate of $1^{\circ} \mathrm{C} / \mathrm{min}$.

\subsection{Interpretation of LTC data}

Normally, the baseline of heat flow during the temperature changing determined by LTC test can be analyzed to calculate the ice content. The baseline will slightly change during the temperature changing, and there will be obvious upwards movement when water transforms into ice and obvious downwards when ice transforms into water. Generally, there are two methods of calculating the baseline and the ice content, called "J-baseline" method (Johannesson, 2010) and "C-baseline" method (Sun \& Scherer, 2010). Both methods can be used for baseline and ice content calculation if due attention is paid $(\mathrm{Wu}$, Johannesson, \& Geiker, 2014b). The "C-baseline" method was employed in this paper.

Since the experimental data were taken at a small temperature interval, $\Delta T$, the mass of ice $m_{c}$ at a certain temperature step can be obtained following the "C-baseline" method,

$$
\begin{aligned}
\mathrm{m}_{C}(T+\Delta T) \approx & m_{C}(T)+\frac{\Delta T}{2}\left(\frac{Q(T)-Q_{0}}{q h_{f}}\right) \\
& \times X+\frac{\Delta T}{2}\left(\frac{Q(T+\Delta T)-Q_{0}}{q h_{f}}\right) * X
\end{aligned}
$$

where $X=\exp \left[-\frac{\left(C_{P C}-C_{P L}\right) \Delta T}{h_{f}}\right]$

$Q_{0}$ represents the heat flow at the point where the water starts to freeze (right before the first peak observed on a measured heat flow curve during freezing), $\mathrm{mW}$; and $\mathrm{Q}(T)$ represents the heat flow at temperature $T$ measured by LTC, mW. $q$ and $h_{f}$ represent the corresponding cooling/heating rate, ${ }^{\circ} \mathrm{C} / \mathrm{s}$, and the heat of fusion, $\mathrm{J} / \mathrm{g} . C_{\mathrm{PC}}$ and $C_{\mathrm{PL}}$ represent the heat capacity of ice and water respectively, $\mathrm{J} /(\mathrm{g} \cdot \mathrm{K})$. It should be noted that the baseline for the freezing and the melting processes almost always are found to be different, due to the hysteresis effect in the freezing and melting process. Equation (2) can also be employed if we analyze the melting process inversely from the high to the low temperature end and treat the data similar to a "freezing process." The $Q_{0}$ would then be the heat flow after all of the ice has been melted (Wu et al., 2014b). Following the work of Sun and Scherer (Sun \& Scherer, 2010), the heat of fusion $(\mathrm{J} / \mathrm{g})$ in the freezing process was adopted as,

$$
h_{f}=332.4
$$

and that in melting process was adopted as,

$$
h_{f}=333.8+1.797\left(T-T_{M}\right)
$$

where $T_{M}$ is the melting temperature of bulk ice. The heat capacity of water and ice are proved to be not very sensitive to temperature; therefore, $4.2176 \mathrm{~J} /(\mathrm{g} \cdot \mathrm{K})$ for water and $2.114 \mathrm{~J} /(\mathrm{g} \cdot \mathrm{K})$ for ice were adopted (Wu et al., 2014b).

After the mass of ice formed or melted in each temperature interval was obtained, the volume of ice $\Delta V \mathrm{c}(\Delta T)$ can be calculated by dividing by the density of ice, $\rho_{c}(T)$ (Lide, 2009),

$$
\Delta V_{\mathrm{c}}(\Delta T)=\frac{\mathrm{m}_{\mathrm{c}}(T+\Delta T)-\mathrm{m}_{\mathrm{c}}(T)}{\rho_{\mathrm{c}}(T)}
$$

and

$$
\rho_{\mathrm{C}}(T) \approx 0.9167-2.053 \times 10^{-4} T-1.357 \times 10^{-6} T^{2}
$$

where $T$ is in degree Celsius. 
There is a unique relation between the pore radius $r_{\mathrm{p}}$ (unit: $\mathrm{nm}$ ) and the temperature during freezing or melting, as follows (Sun \& Scherer, 2010),

$$
\begin{aligned}
& r_{\mathrm{p}}=\frac{64.67}{\Delta T}+0.57 \text { (for freezing) } \\
& r_{\mathrm{p}}=\frac{32.33}{\Delta T}+0.69 \text { (for melting) }
\end{aligned}
$$

From the above relation and the calculated mass of ice at each temperature step, the freezing and melting of water in pores of different sizes can be configured.

\section{RESULTS AND DISCUSSION}

The tested baselines for the freezing and melting processes of cement paste of different degrees of saturation, $100 \%, 93 \%, 89 \%$, and $87 \%$ are given in Figures 1 and 2, respectively. In order to compare the baselines of samples of different degrees of

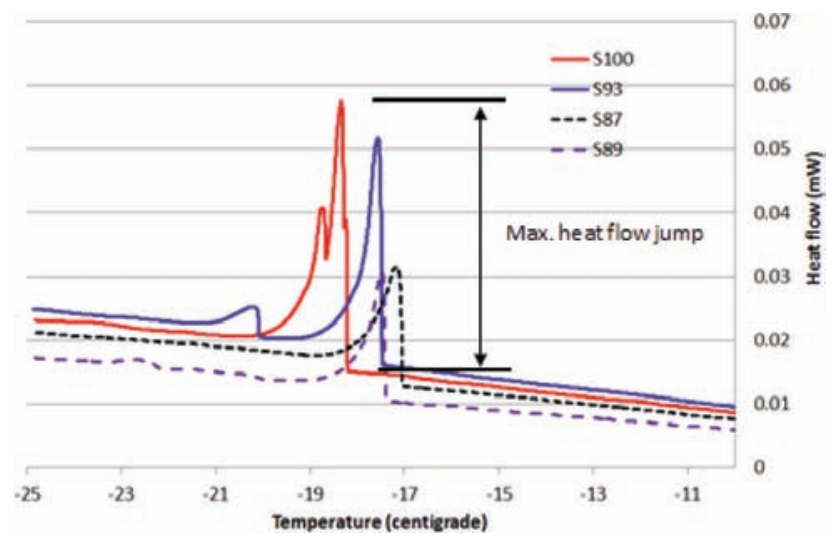

Figure 1. LTC baseline for the freezing process of cement paste of degrees of saturation $(100 \%, 93 \%, 89 \%$, and $87 \%)$.

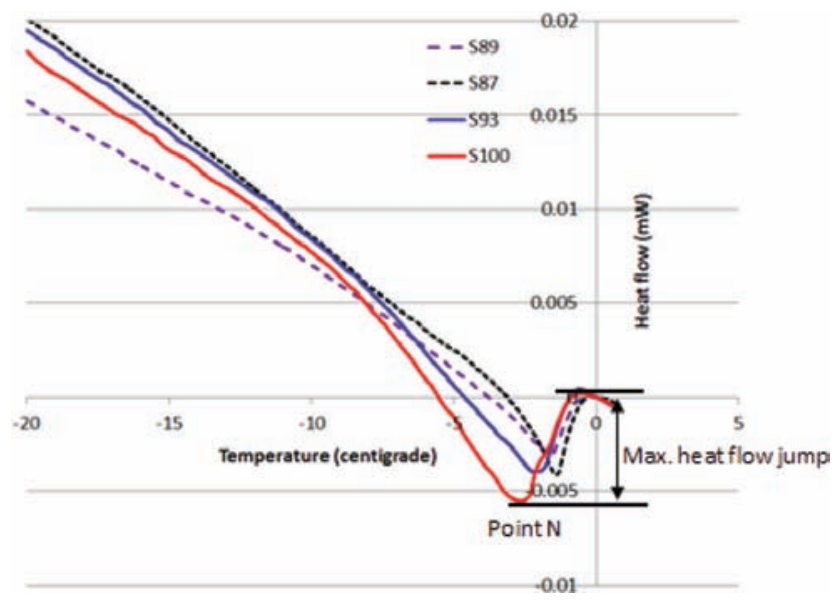

Figure 2. LTC baseline for the melting process of cement paste of different degrees of saturation $(100 \%, 93 \%, 89 \%$, and $87 \%)$. saturation, the baselines are corrected by subtracting a correction parameter, which is adopted as the heat flow at temperature $0, Q(0)$, for each sample. Figure 1 illustrates that the higher the degree of saturation, the larger the maximum heat flow-jump. It indicates that a larger amount of ice is formed after the nucleation point. As for the melting process, the higher the degree of saturation, the larger the maximum heat flow-jump too, but jump downwards, see Figure 2. The point at the trough of the baseline is designated as point $N$.

For the freezing process, ice does not form immediately at the freezing point $T_{\mathrm{M}}$ where bulk ice in the air can be formed. Ice nucleates at a supercooled state, and a larger amount of ice can be formed after nucleation due to the supercooling. Influence of the degree of saturation on the nucleation temperature is shown in Figure 3. The curve in Figure 3 does not show any regularity of the relation between the degree of saturation and the nucleation temperature. Independent of the degree of saturation, the nucleation temperature ranges from $-16.0^{\circ} \mathrm{C}$ to $-19.7^{\circ} \mathrm{C}$ at a rate of $1^{\circ} \mathrm{C} / \mathrm{min}$. For the melting process, dependence of the trough temperature on the degree of saturation is obvious. The trough temperature decreases with the increasing degree of saturation and indicates a linear dependence.

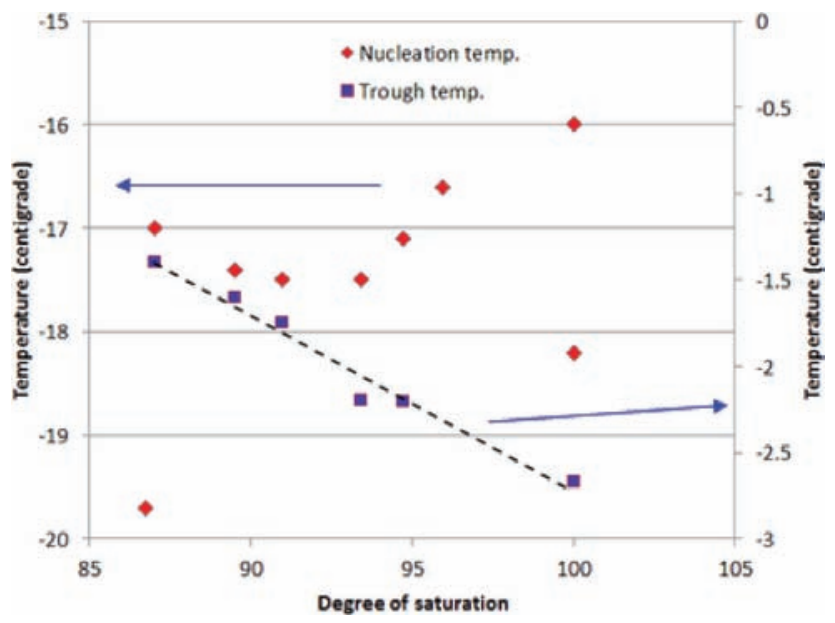

Figure 3. Dependences of nucleation temperature for the freezing process and of the trough temperature for the melting process on the degree of saturation.

According to the "C-baseline" method described above, the ice content at different temperatures and the ice content versus pore size can be obtained, see Figures 4 and 5 . For each sample, the ice content increases with the decreasing temperature during freezing and decreases with the increasing temperature during melting. At a given temperature after nucleation, the ice content of sample with a higher degree of 


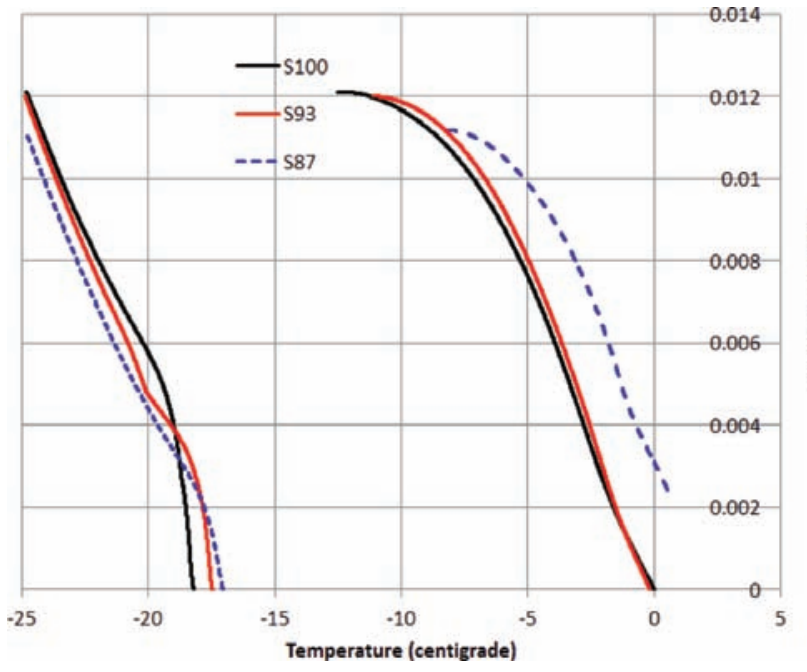

Figure 4. Ice content versus temperature for the samples of different degrees of saturation.

saturation is larger than that with a lower degree of saturation. For the melting process, the melting start temperature, when ice starts to melt, decreases with the increasing degree of saturation. For the sample with the lowest degree of saturation $87 \%$, its melting curve obviously moves to a range with higher temperature comparing to other samples, see Figure 4. It indicates that water redistribution occurs during the freezing and melting process for the unsaturated samples. For the unsaturated samples, before freezing, water evaporates from big pores and liquid water exists in small pores. Theoretically, at nucleation, water in the pores, whose size is larger than the value by Eq. (7), transforms into ice. As the temperature decreases subsequently, the ice grows into smaller pores according to Eq. (7). During melting, when the temperature increases, the ice in the pores, whose size is larger than the value by Eq. (8), transforms into water. Since water evaporates from big pores and liquid water exists in small pores for the unsaturated sample, the freezing and melting curve should show that the ice-water transition occurs in the small pores. However, the ice-to-water transition prefers to take place in big pores, as shown in Figure 5. It indicates that there is water redistribution during a freezing/ melting cycle. That is ice forms in big pores and suck water from small pores as the temperature decreases. For the unsaturated samples, the ice formation may not happen in very small pores, but the water in small pores is sucked into big pores to form ice.

\section{CONCLUSION}

This paper has investigated the freezing and melting process of cement pastes of different degrees of saturation, i.e., $100 \%, 93 \%, 89 \%$, and $87 \%$, using LTC analysis. "C-baseline" method is employed for

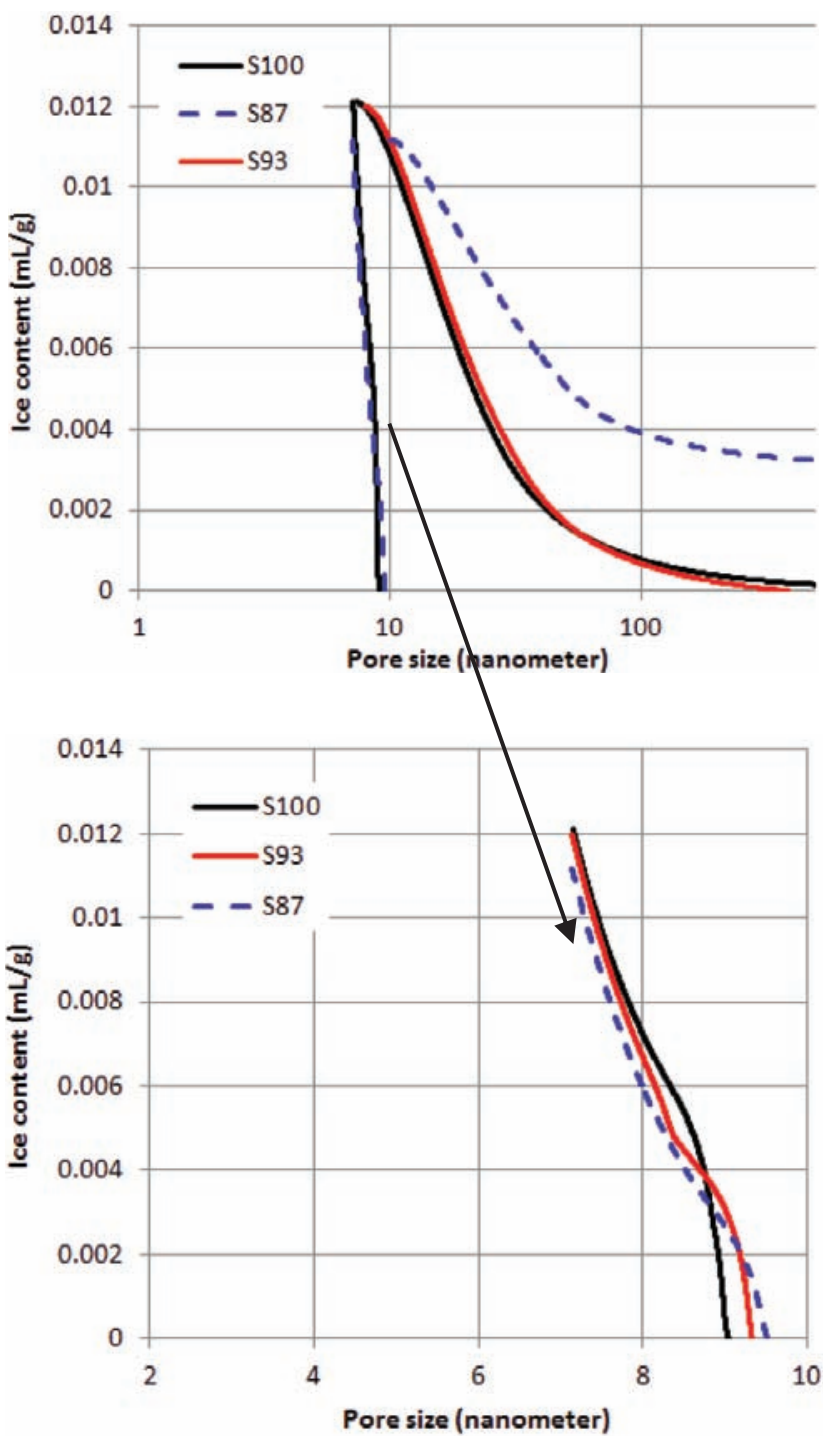

Figure 5. Ice content versus pore size for the samples of different degrees of saturation.

the baseline and ice content calculation. The following conclusions can be addressed from the test:

(1) During the freezing process, the nucleation temperature ranges from $-16.0^{\circ} \mathrm{C}$ to $-19.7^{\circ} \mathrm{C}$ at a rate of $1^{\circ} \mathrm{C} / \mathrm{min}$ cooling/heating and is independent of the degree of saturation.

(2) For the melting process, the trough temperature decreases with the increasing degree of saturation and indicates a linear dependence.

(3) Curves of the ice content versus temperature and of the ice content versus pore size indicate that water movement takes place in unsaturated samples during a freezing/melting cycle. For the unsaturated sample, ice forms in big pores and sucks water from small pores as the temperature decreases, and this leads to a melting stage in big pores compared to the totally saturated one. 


\section{ACKNOWLEDGMENTS}

The financial supports of National Natural Science Foundation of China via Grant No. 51308187 and No. 51461135001, and Natural Science Foundation of Jiangsu Province via Grant No. BK20130837 are greatly acknowledged.

\section{REFERENCES}

Jiang, Z., Li, W., Deng, Z., \& Yan, Z. (2013). Experimental investigation of the factors affecting accuracy and resolution of the pore structure of cement-based materials by thermoporometry. Journal of Zhejiang University - Science A (Applied Physics \& Engineering), 14(10), 720-730. Johannesson, B. (2010). Dimensional and ice content changes of hardened concrete at different freezing and thawing temperatures. Cement and Concrete Composites, 32(1), 73-83.

Lide, D. R. (2009). CRC handbook of chemistry and physics (89th ed.). CRC Press.
Sun, Z., \& Scherer, G. W. (2010). Pore size and shape in mortar by thermoporometry. Cement and Concrete Research, 40, 740-751.

Wang, Z., Zeng, Q., Wang, L., Yao, Y., \& Li, K. (2014). Effect of moisture content on freeze-thaw behavior of cement paste by electrical resistance measurements. Journal of Materials Science, 49, 4305-4314.

Wu, M., \& Johannesson, B. (2014a). Impact of sample saturation on the detected porosity of hardened concrete using low temperature calorimetry. Thermochimica Acta, 580, 66-78.

Wu, M., Johannesson, B., \& Geiker, M. (2014b). Determination of ice content in hardened concrete by low-temperature calorimetry. Journal of Thermal Analysis and Calorimetry, 115, 1335-1351.

Yamamoto, T., Endo, A., Inagi, Y., Ohmori, T., \& Nakaiwa, M. (2005). Evaluation of thermoporometry for characterization of mesoporous materials. Journal of Colloid and Interface Science, 284, 614-620. 